\title{
Localization Operators for Ridgelet Transforms
}

\author{
J. $\mathrm{Li}^{1}, \mathrm{M} . \mathrm{W}$. Wong ${ }^{1 *}$ \\ ${ }^{1}$ Department of Mathematics and Statistics, York University \\ 4700 Keele Street, Toronto, Ontario M3J 1P3, Canada
}

\begin{abstract}
We prove that localization operators associated to ridgelet transforms with $L^{p}$ symbols are bounded linear operators on $L^{2}\left(\mathbb{R}^{n}\right)$. Operators closely related to these localization operators are shown to be in the trace class and a trace formula for them is given.
\end{abstract}

Keywords and phrases: Gabor transforms, wavelet transforms, curvelet transforms, wavelet multipliers, ridgelet transforms, Radon transforms, resolution of the identity formulas, continuous inversion formulas, localization operators, $L^{2}$-boundedness, trace class operators, traces, Lidskii's formula

Mathematics Subject Classification: 42C40, 47G30

\section{Introduction}

For a signal $f$ in $L^{2}(\mathbb{R})$, the Gabor transform or the short-time Fourier transform $G_{\varphi} f$ of $f$ with respect to a window $\varphi$ in $L^{1}(\mathbb{R}) \cap L^{2}(\mathbb{R})$ is defined by

$$
\left(G_{\varphi} f\right)(b, \xi)=(2 \pi)^{-1 / 2} \int_{-\infty}^{\infty} e^{-i x \xi} f(x) \overline{\varphi(x-b)} d x, \quad b, \xi \in \mathbb{R} .
$$

Let us note that

$$
\left(G_{\varphi} f\right)(b, \xi)=(2 \pi)^{-1 / 2}\left(f, M_{\xi} T_{-b} \varphi\right)_{L^{2}(\mathbb{R})}, \quad b, \xi \in \mathbb{R},
$$

where $M_{\xi}$ and $T_{-b}$ are the modulation operator and the translation operator given by

$$
\left(M_{\xi} h\right)(x)=e^{i x \xi} h(x)
$$

and

$$
\left(T_{-b} h\right)(x)=h(x-b)
$$

for all measurable functions $h$ on $\mathbb{R}$ and all $x$ in $\mathbb{R}$. We call the function $M_{\xi} T_{-b} \varphi$ the Gabor wavelet generated from $\varphi$ by translation $T_{-b}$ and modulation $M_{\xi}$.

The usefulness of the Gabor windows in signal analysis is enhanced by the following resolution of the identity formula, which allows the reconstruction of a signal from its Gabor transform.

\footnotetext{
*Corresponding author. E-mail: mwwong@mathstat.yorku.ca This research has been supported by the Natural Sciences and Engineering Research Council of Canada.
} 
Theorem 1.1. Suppose that $\|\varphi\|_{2}=1$, where \|\|$_{2}$ is the norm in $L^{2}(\mathbb{R})$. Then for all $f$ and $g$ in $L^{2}(\mathbb{R})$,

$$
(f, g)_{L^{2}(\mathbb{R})}=(2 \pi)^{-1} \int_{-\infty}^{\infty} \int_{-\infty}^{\infty}\left(f, M_{\xi} T_{-x} \varphi\right)_{L^{2}(\mathbb{R})}\left(M_{\xi} T_{-x} \varphi, g\right)_{L^{2}(\mathbb{R})} d x d \xi
$$

Another way of looking at Theorem 1.1 is that for all $f$ in $L^{2}(\mathbb{R})$,

$$
f=(2 \pi)^{-1} \int_{-\infty}^{\infty} \int_{-\infty}^{\infty}\left(f, M_{\xi} T_{-x} \varphi\right)_{L^{2}(\mathbb{R})} M_{\xi} T_{-x} \varphi d x d \xi
$$

which is also known as a continuous inversion formula for the Gabor transform.

In signal analysis, $\left(G_{\varphi} f\right)(b, \xi)$ gives the time-frequency content of a signal $f$ at time $b$ and frequency $\xi$ by placing the window $\varphi$ at time $b$. The drawback here is that a window of fixed width is used for all time $b$. It is more accurate and desirable if we can have an adaptive window that gives a wide window for low frequency and a narrow window for high frequency. That this can be done comes from familiarity with the wavelet transform that we now recall.

Let $\varphi \in L^{2}(\mathbb{R})$ be such that

$$
\int_{-\infty}^{\infty} \frac{|\hat{\varphi}(\xi)|^{2}}{|\xi|} d \xi<\infty
$$

where $\hat{\varphi}$ is the Fourier transform of $\varphi$. The convention that we use in this paper is that the Fourier transform $\hat{f}$ of a function $f$ in $L^{1}\left(\mathbb{R}^{n}\right)$ is defined by

$$
\hat{f}(\xi)=(2 \pi)^{-n / 2} \int_{\mathbb{R}^{n}} e^{-i x \cdot \xi} f(x) d x, \quad \xi \in \mathbb{R}^{n} .
$$

Such a function $\varphi$ is said to satisfy the admissibility condition and is sometimes called the mother affine wavelet. The adjective affine comes from the connection with the affine group that is the underpinning of the wavelet transforms. See Chapter 18 of [19] in this connection.

Let $\varphi \in L^{2}(\mathbb{R})$ be a mother affine wavelet. Then for all $b$ in $\mathbb{R}$ and $a$ in $\mathbb{R} \backslash\{0\}$, we define the affine wavelet $\varphi_{b, a}$ by

$$
\varphi_{b, a}(x)=\frac{1}{\sqrt{|a|}} \varphi\left(\frac{x-b}{a}\right), \quad x \in \mathbb{R} .
$$

We note that $\varphi_{b, a}$ is generated from the function $\varphi$ by translation and dilation. To put things in perspective, let $b \in \mathbb{R}$ and let $a \in \mathbb{R} \backslash\{0\}$. Then we see that the wavelet $\varphi_{b, a}$ can be expressed as

$$
\varphi_{b, a}=T_{-b} D_{1 / a} \varphi
$$

where $D_{1 / a}$ is the dilation operator defined by

$$
\left(D_{1 / a} h\right)(x)=\frac{1}{\sqrt{|a|}} h\left(\frac{x}{a}\right)
$$

for all measurable functions $h$ on $\mathbb{R}$ and all $x$ in $\mathbb{R}$.

Let $\varphi$ be a mother affine wavelet. Then the wavelet transform $\Omega_{\varphi} f$ of a function $f$ in $L^{2}(\mathbb{R})$ is defined to be the function on $\mathbb{R} \times(\mathbb{R} \backslash\{0\})$ by

$$
\left(\Omega_{\varphi} f\right)(b, a)=\left(f, \varphi_{b, a}\right)_{L^{2}(\mathbb{R})}
$$

for all $b$ in $\mathbb{R}$ and $a$ in $\mathbb{R} \backslash\{0\}$. The analysis of the wavelet transform is based on the following resolution of the identity formula, which is also a continuous inversion formula. 
Theorem 1.2. Let $\varphi$ be a mother affine wavelet. Then for all functions $f$ and $g$ in $L^{2}(\mathbb{R})$,

$$
(f, g)_{L^{2}(\mathbb{R})}=\frac{1}{c_{\varphi}} \int_{-\infty}^{\infty} \int_{-\infty}^{\infty}\left(f, \varphi_{b, a}\right)_{L^{2}(\mathbb{R})}\left(\varphi_{b, a}, g\right)_{L^{2}(\mathbb{R})} \frac{d b d a}{a^{2}}
$$

where

$$
c_{\varphi}=2 \pi \int_{-\infty}^{\infty} \frac{|\hat{\varphi}(\xi)|^{2}}{|\xi|} d \xi
$$

Remark 1.3. It can also be proved that a necessary condition for the continuous inversion formula to hold is that $\varphi$ has to be a mother affine wavelet. Indeed, suppose that $\int_{-\infty}^{\infty} \int_{-\infty}^{\infty}\left(f, \varphi_{b, a}\right)_{L^{2}(\mathbb{R})}\left(\varphi_{b, a}, g\right)_{L^{2}(\mathbb{R})} \frac{d b d a}{a^{2}}$ exists for all $f$ and $g$ in $L^{2}(\mathbb{R})$. Then, letting $f=g=\varphi$, we get

$$
\int_{-\infty}^{\infty} \int_{-\infty}^{\infty}\left|\left(\varphi, \varphi_{b, a}\right)_{L^{2}(\mathbb{R})}\right|^{2} \frac{d b d a}{a^{2}}<\infty
$$

which can be shown to be the same as

$$
\int_{-\infty}^{\infty} \frac{|\hat{\varphi}(\xi)|^{2}}{|\xi|} d \xi<\infty
$$

The resolution of the identity formula leads to the reconstruction formula, which says that

$$
f=\frac{1}{c_{\varphi}} \int_{-\infty}^{\infty} \int_{-\infty}^{\infty}\left(f, \varphi_{b, a}\right)_{L^{2}(\mathbb{R})} \varphi_{b, a} \frac{d b d a}{a^{2}}
$$

for all $f$ in $L^{2}(\mathbb{R})$. In other words, we have a continuous inversion formula for the signal $f$ from a knowledge of its time-scale spectrum.

As in the case of the Gabor transform, there is a window $\varphi_{b, a}$ in the wavelet transform. Unlike the case of the Gabor transform, the window $\varphi_{b, a}$ is adjustable in the sense that it is narrow if the scale $a$ is small and wide if the scale $a$ is big.

Details on the analysis and applications of wavelets can be found in $[6,8,19]$.

The wavelet transforms as defined are essentially one-dimensional time-frequency tools since the frequency $\xi$ and scale $a$ can be thought of as being related by $a=1 / \xi$.

Another multiscale integral transform, which is dubbed the curvelet transform [3], has emerged in time-frequency analysis. It is an interesting fact that the resolution of the identity formula is now only valid for high-frequency signals. The full resolution of the identity formula for all signals with finite energy requires an additional term to cope with low frequency signals as well. This additional term turns out to be a wavelet multiplier first studied systematically in $[9,19]$.

Closely related to multi-dimensional wavelet transforms are ridgelet transforms first introduded by Emmanuel Candés in his 1998 Ph.D. thesis [1,2]. As a matter of historical fact, ridgelets predate curvelets. To recall, let $\Psi: \mathbb{R} \rightarrow \mathbb{R}$ be a Schwartz function such that the admissibility condition

$$
\int_{-\infty}^{\infty} \frac{|\hat{\Psi}(\xi)|^{2}}{|\xi|^{n}} d \xi<\infty
$$

holds. Such a function $\Psi$ is appropriately called a mother ridgelet. The phase space $\Gamma$ that is relevant to the ridgelet transforms is given by

$$
\Gamma=(0, \infty) \times \mathbb{S}^{n-1} \times \mathbb{R}
$$

where $\mathbb{S}^{n-1}$ is the unit sphere centered at the origin in $\mathbb{R}^{n}$. Points in $\Gamma$ are sometimes denoted by $\gamma$ and the measure $d \gamma$ on $\Gamma$ is given by

$$
d \gamma=\frac{d a}{a^{n+1}} d u d b
$$


where $d u$ is the surface measure of $\mathbb{S}^{n-1}$. For $\gamma=(a, u, b) \in \Gamma$, the ridgelet $\Psi_{\gamma}$ corresponding to $\gamma$ is defined by

$$
\Psi_{\gamma}(x)=\frac{1}{\sqrt{a}} \Psi\left(\frac{(u \cdot x)-b}{a}\right), \quad x \in \mathbb{R}^{n} .
$$

Let $\Psi$ be a mother ridgelet. Then we define the ridgelet transform $R_{\Psi} f$ of a function $f$ in $L^{2}\left(\mathbb{R}^{n}\right)$ to be the function on $\Gamma$ by

$$
\left(R_{\Psi} f\right)(a, u, b)=\left(\Psi_{a, u, b}, f\right)_{L^{2}\left(\mathbb{R}^{n}\right)}, \quad(a, u, b) \in \Gamma .
$$

The following resolution of the identity formula for ridgelet transforms can be found in [1].

Theorem 1.4. Let $\Psi$ be a mother ridgelet and let $K_{\Psi}$ be the number given by

$$
K_{\Psi}=\int_{-\infty}^{\infty} \frac{|\hat{\Psi}(\xi)|^{2}}{|\xi|^{n}} d \xi
$$

Then for all $f$ and $g$ in $L^{2}\left(\mathbb{R}^{n}\right)$,

$$
(f, g)_{L^{2}\left(\mathbb{R}^{n}\right)}=c_{\Psi} \int_{\Gamma}\left(f, \Psi_{\gamma}\right)_{L^{2}\left(\mathbb{R}^{n}\right)}\left(\Psi_{\gamma}, g\right)_{L^{2}\left(\mathbb{R}^{n}\right)} d \gamma
$$

where

$$
c_{\Psi}=(2 \pi)^{-n} K_{\Psi}^{-1} .
$$

With the resolution of the identity formulas for ridgelet transforms in place, a symbol $\tau$ defined on the phase space can be inserted into the integral in the resolution of the identity formula for the purpose of localizing and a localization operator $L_{\tau}$ for the corresponding transform is obtained. Precisely, let $\tau$ be a suitable measurable function on $\Gamma$. Then for every function $f$ in $L^{2}\left(\mathbb{R}^{n}\right)$, we define $L_{\tau} f$ to be the function in $L^{2}\left(\mathbb{R}^{n}\right)$ by

$$
\left(L_{\tau} f, g\right)_{L^{2}\left(\mathbb{R}^{n}\right)}=c_{\Psi} \int_{\Gamma} \tau(\gamma)\left(f, \Psi_{\gamma}\right)_{L^{2}\left(\mathbb{R}^{n}\right)}\left(\Psi_{\gamma}, g\right)_{L^{2}\left(\mathbb{R}^{n}\right)} d \gamma
$$

for all functions $g$ in $L^{2}\left(\mathbb{R}^{n}\right)$.

The aim of this paper is to give conditions on the symbols to ensure that the corresponding localization operators are bounded linear operators on $L^{2}\left(\mathbb{R}^{n}\right)$, and to guarantee that related localization operators are in the trace class $S_{1}$. A trace formula for these trace class operators is given. We first give a recall of the Radon transform that we need in the study of the ridgelet transforms in Section 2. In Section 3, we give conditions on the symbols to guarantee that the corresponding localization operators are bounded linear operators on $L^{2}\left(\mathbb{R}^{n}\right)$. Localization operators in the trace class $S_{1}$ together with a trace formula, are given in Section 4. The non-self-adjoint operators with trace given in this paper are reminiscent of the Landau-Pollak-Slepian operators [10,13-16] and wavelet multipliers $[4,7,9,21,22]$. Chapters 19 and 20 of [19] contain self-contained contents on, respectively, the wavelet multipliers and the Landau-PollakSlepian operators.

\section{The Radon Transform}

We give a recall of the Radon transform that we need in this paper. Let $u \in \mathbb{S}^{n-1}$. Let $s_{1}, s_{2}, \ldots, s_{n-1}$ be unit vectors in $\mathbb{R}^{n}$ such that $\left\{s_{1}, \ldots, s_{n-1}, u\right\}$ is an orthonormal basis for $\mathbb{R}^{n}$. Then for all points $x$ in $\mathbb{R}^{n}$, we can write

$$
x=t u+\sum_{j=1}^{n-1} v_{j} s_{j},
$$


where $t, v_{1}, \ldots, v_{n-1}$ are real numbers. If $f$ is a Schwartz function on $\mathbb{R}^{n}$, then we define the Radon transform of $f$ in the direction $u$ by

$$
\left(R_{u} f\right)(t)=\int_{\mathbb{R}^{n-1}} f\left(t u+\sum_{j=1}^{n-1} v_{j} s_{j}\right) d v_{1} d v_{2} \cdots d v_{n-1},
$$

It can be proved that for each $u$ in $\mathbb{S}^{n-1}$, the definition of $R_{u} f$ is independent of the choice of $s_{1}, s_{2}, \ldots, s_{n-1}$ and

$$
\int_{\mathbb{R}^{n}} f(x) d x=\int_{-\infty}^{\infty}\left(R_{u} f\right)(t) d t
$$

Furthermore, we have the following result.

Theorem 2.1. Let $f$ be a Schwartz function on $\mathbb{R}^{n}$. Then for each $u$ in $\mathbb{S}^{n-1}, R_{u} f$ is a Schwartz function on $\mathbb{R}$ and

$$
\widehat{R_{u} f}(\xi)=(2 \pi)^{(n-1) / 2} \hat{f}(\xi u), \quad \xi \in \mathbb{R} .
$$

Section 5.2 of Chapter 6 in [17] contains more details on the Radon transform and its properties.

\section{3. $L^{2}$-Boundedness of Localization Operators for Ridgelet Transforms}

We can now give the main result on the $L^{2}$-boundedness of localization operators for ridgelet transforms.

Theorem 3.1. Let $\tau \in L^{p}(\Gamma), 1 \leq p \leq \infty$. Then the localization operator $L_{\tau}: L^{2}\left(\mathbb{R}^{n}\right) \rightarrow L^{2}\left(\mathbb{R}^{n}\right)$ with symbol $\tau$ is a bounded linear operator. Moreover,

$$
\left\|L_{\tau}\right\|_{*} \leq\left((2 \pi)^{n-1} c_{\Psi}\|\Psi\|_{L^{2}(\mathbb{R})}^{2}\right)^{1 / p}\|\tau\|_{L^{p}(\Gamma)},
$$

where \|\|$_{*}$ is the norm in the $C^{*}$-algebra of all bounded linear operators on $L^{2}\left(\mathbb{R}^{n}\right)$.

Proof. Let $\tau \in L^{\infty}(\Gamma)$. Then using the Schwarz inequality and the resolution of the identity formula for ridgelet transforms, we have for all $f$ and $g$ in $L^{2}\left(\mathbb{R}^{n}\right)$

$$
\begin{aligned}
& \left|\left(L_{\tau} f, g\right)_{L^{2}\left(\mathbb{R}^{n}\right)}\right| \\
= & \left|c_{\Psi} \int_{\Gamma} \tau(\gamma)\left(f, \Psi_{\gamma}\right)_{L^{2}\left(\mathbb{R}^{n}\right)}\left(\Psi_{\gamma}, g\right)_{L^{2}\left(\mathbb{R}^{n}\right)} d \gamma\right| \\
\leq & c_{\Psi} \int_{\Gamma}|\tau(\gamma)|\left|\left(f, \Psi_{\gamma}\right)_{L^{2}\left(\mathbb{R}^{n}\right)}\left(\Psi_{\gamma}, g\right)_{L^{2}\left(\mathbb{R}^{n}\right)}\right| d \gamma \\
\leq & c_{\Psi}\|\tau\|_{L^{\infty}(\Gamma)} \int_{\Gamma}\left|\left(f, \Psi_{\gamma}\right)_{L^{2}\left(\mathbb{R}^{n}\right)}\right|\left|\left(\Psi_{\gamma}, g\right)_{L^{2}\left(\mathbb{R}^{n}\right)}\right| d \gamma \\
\leq & c_{\Psi}\|\tau\|_{L^{\infty}(\Gamma)}\left\{\int_{\Gamma}\left|\left(f, \Psi_{\gamma}\right)_{L^{2}\left(\mathbb{R}^{n}\right)}\right|^{2} d \gamma\right\}^{1 / 2}\left\{\int_{\Gamma}\left|\left(\Psi_{\gamma}, g\right)_{L^{2}\left(\mathbb{R}^{n}\right)}\right|^{2} d \gamma\right\}^{1 / 2} \\
= & c_{\Psi}\|\tau\|_{L^{\infty}(\Gamma)}\left\{\frac{\|f\|_{L^{2}\left(\mathbb{R}^{n}\right)}^{2}}{c_{\Psi}}\right\}^{1 / 2}\left\{\frac{\|g\|_{L^{2}\left(\mathbb{R}^{n}\right)}^{2}}{c_{\Psi}}\right\}^{1 / 2} \\
= & \|\tau\|_{L^{\infty}(\Gamma)}\|f\|_{L^{2}\left(\mathbb{R}^{n}\right)}\|g\|_{L^{2}\left(\mathbb{R}^{n}\right)} .
\end{aligned}
$$

Now, let $\tau \in L^{1}(\Gamma)$. Then for all functions $f$ and $g$ in $L^{2}\left(\mathbb{R}^{n}\right)$,

$$
\begin{aligned}
& \left|\left(L_{\tau} f, g\right)_{L^{2}\left(\mathbb{R}^{n}\right)}\right| \\
= & \left|c_{\Psi} \int_{\Gamma} \tau(\gamma)\left(f, \Psi_{\gamma}\right)_{L^{2}\left(\mathbb{R}^{n}\right)}\left(\Psi_{\gamma}, g\right)_{L^{2}\left(\mathbb{R}^{n}\right)} d \gamma\right| \\
\leq & c_{\Psi} \int_{\Gamma}|\tau(\gamma)|\left|\left(f, \Psi_{\gamma}\right)_{L^{2}\left(\mathbb{R}^{n}\right)}\right|\left|\left(\Psi_{\gamma}, g\right)_{L^{2}\left(\mathbb{R}^{n}\right)}\right| d \gamma .
\end{aligned}
$$


For $\gamma=(a, u, b)$, we let $\Psi_{a}$ be the function on $\mathbb{R}$ defined by

$$
\Psi_{a}(y)=a^{-1 / 2} \Psi(y / a), \quad y \in \mathbb{R},
$$

and we get

$$
\begin{aligned}
& \left(f, \Psi_{\gamma}\right)_{L^{2}\left(\mathbb{R}^{n}\right)} \\
= & \int_{\mathbb{R}^{n}} f(x) \frac{1}{\sqrt{a}} \Psi_{a^{-1}}((u \cdot x)-b) d x \\
= & \int_{-\infty}^{\infty} \int_{\mathbb{R}^{n-1}} f\left(t u+\sum_{j=1}^{n-1} v_{j} s_{j}\right) \frac{1}{\sqrt{a}} \Psi_{a^{-1}}\left(u \cdot\left(t u+\sum_{j=1}^{n-1} v_{j} s_{j}\right)-b\right) d v d t \\
= & \int_{-\infty}^{\infty} \int_{\mathbb{R}^{n-1}} f\left(t u+\sum_{j=1}^{n-1} v_{j} s_{j}\right) \frac{1}{\sqrt{a}} \Psi_{a^{-1}}(t-b) d v d t \\
= & \int_{-\infty}^{\infty} \frac{1}{\sqrt{a}} \Psi_{a^{-1}}(t-b)\left\{\int_{\mathbb{R}^{n-1}} f\left(t u+\sum_{j=1}^{n-1} v_{j} s_{j}\right) d v\right\} d t \\
= & \int_{-\infty}^{\infty} \frac{1}{\sqrt{a}} \Psi_{-a^{-1}}(b-t)\left(R_{u} f\right)(t) d t \\
= & \frac{1}{\sqrt{a}}\left(\Psi_{-a^{-1}} *\left(R_{u} f\right)\right)(b) .
\end{aligned}
$$

Since

$$
\left\|\frac{1}{\sqrt{a}} \Psi_{a^{-1}}\right\|_{L^{2}(\mathbb{R})}=\|\Psi\|_{L^{2}(\mathbb{R})},
$$

it follows from (3.2)-(3.4) and the Schwarz inequality that

$$
\begin{aligned}
& \left|\left(L_{\tau} f, g\right)_{L^{2}\left(\mathbb{R}^{n}\right)}\right| \\
\leq & c_{\Psi} \int_{-\infty}^{\infty} \int_{\mathbb{S}^{n-1}} \int_{0}^{\infty}|\tau(a, u, b)| \\
\qquad & \left|\left(\frac{1}{\sqrt{a}} \Psi_{-a^{-1}} *\left(R_{u} f\right)\right)(b)\right| \\
= & \left.c_{\Psi} \int_{-\infty}^{\infty} \int_{\mathbb{S}^{n-1}} \int_{0}^{\infty} \mid \tau\left(R_{u} g\right)\right)(b) \mid \frac{d a}{a^{n+1}} d u d b \\
= & c_{\Psi}\|\Psi\|_{L^{2}(\mathbb{R})}^{2} \int_{-\infty}^{\infty} \int_{\mathbb{S}^{n-1}} \int_{0}^{\infty}|\tau(a, u, b)|\left\|R_{u} f\right\|_{L^{2}(\mathbb{R})}\left\|R_{u} g\right\|_{L^{2}(\mathbb{R})} \frac{d a}{a^{n+1}} d u d b .
\end{aligned}
$$

For all Schwartz functions $f$ on $\mathbb{R}^{n}$, we get by Plancherel's formula

$$
\left\|R_{u} f\right\|_{L^{2}(\mathbb{R})}^{2}=\left\|\widehat{R_{u} f}\right\|_{L^{2}(\mathbb{R})}^{2} .
$$

Now, we note that the restriction theorem of the Fourier transform holds to the effect that

$$
\int_{-\infty}^{\infty}|\hat{f}(\xi u)|^{2} d \xi \leq \int_{\mathbb{R}^{n}}|\hat{f}(\eta)|^{2} d \eta=\int_{\mathbb{R}^{n}}|f(x)|^{2} d x .
$$

Indeed,

$$
\int_{\mathbb{R}^{n}}|\hat{f}(\eta)|^{2} d \eta=\int_{\mathbb{R}^{n-1}} \int_{-\infty}^{\infty}\left|\hat{f}\left(\xi u+\sum_{j=1}^{\infty} v_{j} s_{j}\right)\right|^{2} d \xi d v .
$$


Then there exists a vector $v$ in $\mathbb{R}^{n-1}$ such that

$$
\int_{-\infty}^{\infty}\left|\hat{f}\left(\xi u+\sum_{j=1}^{n-1} v_{j} s_{j}\right)\right|^{2} d \xi \leq \int_{\mathbb{R}^{n}}|\hat{f}(\eta)|^{2} d \eta .
$$

For if this is not true, then for all $v \in \mathbb{R}^{n-1}$,

$$
\int_{-\infty}^{\infty}\left|\hat{f}\left(\xi u+\sum_{j=1}^{n-1} v_{j} s_{j}\right)\right|^{2} d \xi>\int_{\mathbb{R}^{n}}|\hat{f}(\eta)|^{2} d \eta
$$

and hence

$$
\begin{aligned}
\int_{\mathbb{R}^{n}}|\hat{f}(\eta)|^{2} d \eta & =\int_{\mathbb{R}^{n-1}} \int_{-\infty}^{\infty}\left|\hat{f}\left(\xi u+\sum_{j=1}^{n-1} v_{j} s_{j}\right)\right|^{2} d \xi d v \\
& >\int_{\mathbb{R}^{n-1}} \int_{\mathbb{R}^{n}}|\hat{f}(\eta)|^{2} d \eta d v=\infty
\end{aligned}
$$

which is a contradiction. So, there exists a $v$ in $\mathbb{R}^{n-1}$ such that

$$
\int_{-\infty}^{\infty}\left|\left(M_{-\sum_{j=1}^{n-1} v_{j} s_{j}} f\right)^{\wedge}(\xi u)\right|^{2} d \xi \leq \int_{\mathbb{R}^{n}}|\hat{f}(\eta)|^{2} d \eta=\int_{\mathbb{R}^{n}}|f(x)|^{2} d x
$$

and hence

$$
\int_{-\infty}^{\infty}|\hat{f}(\xi u)|^{2} d \xi \leq \int_{\mathbb{R}^{n}}\left|\left(M_{\sum_{j=1}^{n-1} v_{j} s_{j}} f\right)(x)\right|^{2} d x=\int_{\mathbb{R}^{n}}|f(x)|^{2} d x
$$

where

$$
\left(M_{ \pm \sum_{j=1}^{n-1} v_{j} s_{j}} f\right)(x)=e^{ \pm i x \cdot \sum_{j=1}^{n-1} v_{j} s_{j}} f(x), \quad x \in \mathbb{R}^{n} .
$$

By Theorem 2.1 and (3.7),

$$
\begin{aligned}
\left\|R_{u} f\right\|_{L^{2}(\mathbb{R})}^{2} & =\int_{-\infty}^{\infty}\left|\widehat{R_{u} f}(\xi)\right|^{2} d \xi \\
& =(2 \pi)^{n-1} \int_{-\infty}^{\infty}|\hat{f}(\xi u)|^{2} d \xi \\
& \leq(2 \pi)^{n-1}\|\hat{f}\|_{L^{2}\left(\mathbb{R}^{n}\right)}^{2} \\
& =(2 \pi)^{n-1}\|f\|_{L^{2}\left(\mathbb{R}^{n}\right)}
\end{aligned}
$$

Thus, by (3.5) and (3.8),

$$
\left|\left(L_{\tau} f, g\right)_{L^{2}(\mathbb{R})}\right| \leq(2 \pi)^{n-1} c_{\Psi}\|\Psi\|_{L^{2}(\mathbb{R})}^{2}\|f\|_{L^{2}\left(\mathbb{R}^{n}\right)}\|g\|_{L^{2}\left(\mathbb{R}^{n}\right)}\|\tau\|_{L^{1}(\Gamma)}
$$

and hence

$$
\left\|L_{\tau} f\right\|_{L^{2}\left(\mathbb{R}^{n}\right)} \leq(2 \pi)^{n-1} c_{\Psi}\|\Psi\|_{L^{2}(\mathbb{R})}^{2}\|\tau\|_{L^{1}(\Gamma)}\|f\|_{L^{2}\left(\mathbb{R}^{n}\right)} .
$$

Using the Riesz-Thorin theorem [18], we get

$$
\begin{aligned}
\left\|L_{\tau} f\right\|_{L^{2}\left(\mathbb{R}^{n}\right)} & \leq\left((2 \pi)^{n-1} c_{\Psi}\|\Psi\|_{L^{2}(\mathbb{R})}^{2}\|f\|_{L^{2}\left(\mathbb{R}^{n}\right)}\right)^{1 / p}\|f\|_{L^{2}\left(\mathbb{R}^{n}\right)}^{1-(1 / p)}\|\tau\|_{L^{p}(\Gamma)} \\
& =\left((2 \pi)^{n-1} c_{\Psi}\|\Psi\|_{L^{2}(\mathbb{R})}^{2}\right)^{1 / p}\|\tau\|_{L^{p}(\Gamma)}\|f\|_{L^{2}\left(\mathbb{R}^{n}\right)}
\end{aligned}
$$

and the proof is complete. 


\section{Trace Class Localization Operators}

For the sake of self-containedness, we give a brief recall of the basic results on trace class operators and traces in $[19,20]$. Let $A$ be a compact operator on a complex, separable and infinite-dimensional Hilbert space $X$. Then $\left(A^{*} A\right)^{1 / 2}$ is a compact and positive operator on $X$, where $A^{*}$ is the adjoint of $A$. Let $\left\{\varphi_{k}: k=1,2, \ldots\right\}$ be an orthonormal basis of $X$ consisting of eigenvectors of $A$, and for $k=1,2, \ldots$, let $s_{k}$ be the eigenvalue of $\left(A^{*} A\right)^{1 / 2}$ corresponding to the eigenvector $\varphi_{k}$. Then we say that $A$ is in the trace class $S_{1}$ if

$$
\sum_{k=1}^{\infty} s_{k}<\infty .
$$

If $A$ is in $S_{1}$, then for all orthonormal bases $\left\{\varphi_{k}: k=1,2, \ldots\right\}$ of $X$, the series $\sum_{k=1}^{\infty}\left(A \varphi_{k}, \varphi_{k}\right)$ is absolutely convergent and the sum is independent of the choice of the orthonormal basis $\left\{\varphi_{k}: k=1,2, \ldots\right\}$ for $X$. We define the trace $\operatorname{tr}(A)$ by

$$
\operatorname{tr}(A)=\sum_{k=1}^{\infty}\left(A \varphi_{k}, \varphi_{k}\right),
$$

where $\left\{\varphi_{k}: k=1,2, \ldots\right\}$ is any orthonormal basis for $X$. It is a well-known result of Lidskii [12] that the trace $\operatorname{tr}(A)$ of a trace class operator $A$ is the sum of the eigenvalues of $A$, where the multiplicity of each eigenvalue is taken into account. The following results can be found in [19].

Theorem 4.1. Let $A: X \rightarrow X$ be a positive operator such that

$$
\sum_{k=1}^{\infty}\left(A \varphi_{k}, \varphi_{k}\right)<\infty
$$

for all orthonormal bases $\left\{\varphi_{k}: k=1,2, \ldots\right\}$ of $X$, where $($,$) is the inner product in X$. Then $A \in S_{1}$.

The following result is an immediate consequence of Theorem 3.1.

Theorem 4.2. Let $\tau \in L^{p}(\Gamma), 1 \leq p \leq \infty$. Then for all functions $\varphi$ in the Schwartz space $\mathcal{S}$, the localization operator $\varphi L_{\tau} \bar{\varphi}: L^{2}\left(\mathbb{R}^{n}\right) \rightarrow L^{2}\left(\mathbb{R}^{n}\right)$ is a bounded linear operator and

$$
\left\|\varphi L_{\tau} \bar{\varphi}\right\|_{*} \leq\|\varphi\|_{L^{\infty}\left(\mathbb{R}^{n}\right)}^{2}\left((2 \pi)^{n-1} c_{\Psi}\|\Psi\|_{L^{2}(\mathbb{R})}^{2}\right)^{1 / p}\|\tau\|_{L^{p}(\Gamma)} .
$$

Theorem 4.3. Let $\tau / a \in L^{1}(\Gamma)$. Then for all functions $\varphi$ in $\mathcal{S}$, the localization operator $\varphi L_{\tau} \bar{\varphi}$ : $L^{2}\left(\mathbb{R}^{n}\right) \rightarrow L^{2}\left(\mathbb{R}^{n}\right)$ is a trace class operator and

$$
\operatorname{tr}\left(\varphi L_{\tau} \bar{\varphi}\right)=c_{\Psi} \int_{\Gamma} \tau(\gamma)\left\|\varphi \Psi_{\gamma}\right\|_{L^{2}\left(\mathbb{R}^{n}\right)}^{2} d \gamma
$$

Proof. We first assume that $\tau$ is a nonnegative real-valued function. Then for all functions $f$ in $L^{2}\left(\mathbb{R}^{n}\right)$,

$$
\begin{aligned}
& \left(\varphi L_{\tau} \bar{\varphi} f, f\right)_{L^{2}\left(\mathbb{R}^{n}\right)} \\
= & \left(L_{\tau} \bar{\varphi} f, \bar{\varphi} f\right)_{L^{2}\left(\mathbb{R}^{n}\right)} \\
= & c_{\Psi} \int_{\Gamma} \tau(\gamma)\left|\left(\bar{\varphi} f, \Psi_{\gamma}\right)_{L^{2}\left(\mathbb{R}^{n}\right)}\right|^{2} d \gamma \\
= & c_{\Psi} \int_{\Gamma} \tau(\gamma)\left|\left(f, \varphi \Psi_{\gamma}\right)_{L^{2}\left(\mathbb{R}^{n}\right)}\right|^{2} d \gamma .
\end{aligned}
$$


Since for $\gamma=(a, u, b)$,

$$
\begin{aligned}
& \left|\left(f, \varphi \Psi_{\gamma}\right)_{L^{2}\left(\mathbb{R}^{n}\right)}\right|^{2} \\
= & \left|\int_{\mathbb{R}^{n}} f(x) \bar{\varphi}(x) \frac{1}{\sqrt{a}} \Psi\left(\frac{(u \cdot x)-b}{a}\right) d x\right|^{2} \\
= & \frac{1}{a}\left|\int_{-\infty}^{\infty} \int_{\mathbb{R}^{n-1}} f\left(t u+\sum_{j=1}^{n-1} v_{j} s_{j}\right) \bar{\varphi}\left(t u+\sum_{j=1}^{n-1} v_{j} s_{j}\right) \Psi\left(\frac{t-b}{a}\right) d v d t\right|^{2} \\
\leq & \frac{1}{a}\|\Psi\|_{L^{\infty}(\mathbb{R})}^{2}\left(\int_{-\infty}^{\infty} \int_{\mathbb{R}^{n-1}}\left|f\left(t u+\sum_{j=1}^{n-1} v_{j} s_{j}\right) \bar{\varphi}\left(t u+\sum_{j=1}^{n-1} v_{j} s_{j}\right)\right| d v d t\right)^{2} \\
= & \frac{1}{a}\|\Psi\|_{L^{\infty}(\mathbb{R})}^{2}\left(\int_{\mathbb{R}^{n}}|f(x) \bar{\varphi}(x)| d x\right)^{2} \\
\leq & \frac{1}{a}\|\Psi\|_{L^{\infty}(\mathbb{R})}^{2}\|f\|_{L^{2}\left(\mathbb{R}^{n}\right)}^{2}\|\varphi\|_{L^{2}\left(\mathbb{R}^{n}\right)}^{2} .
\end{aligned}
$$

Therefore

$$
\left(\varphi L_{\tau} \bar{\varphi} f, f\right)_{L^{2}\left(\mathbb{R}^{n}\right)} \geq 0, \quad f \in L^{2}\left(\mathbb{R}^{n}\right) .
$$

Thus, $\varphi L_{\tau} \bar{\varphi}: L^{2}\left(\mathbb{R}^{n}\right) \rightarrow L^{2}\left(\mathbb{R}^{n}\right)$ is a positive operator. Now, let $\left\{\varphi_{k}: k=1,2, \ldots\right\}$ be an orthonormal basis for $L^{2}\left(\mathbb{R}^{n}\right)$. Then

$$
\begin{aligned}
& \sum_{k=1}^{\infty}\left(\varphi L_{\tau} \bar{\varphi} \varphi_{k}, \varphi_{k}\right)_{L^{2}\left(\mathbb{R}^{n}\right)} \\
= & \sum_{k=1}^{\infty}\left(L_{\tau} \bar{\varphi} \varphi_{k}, \bar{\varphi} \varphi_{k}\right)_{L^{2}\left(\mathbb{R}^{n}\right)} \\
= & c_{\Psi} \int_{\Gamma} \tau(\gamma) \sum_{k=1}^{\infty}\left|\left(\bar{\varphi} \varphi_{k}, \Psi_{\gamma}\right)_{L^{2}\left(\mathbb{R}^{n}\right)}\right|^{2} d \gamma \\
= & c_{\Psi} \int_{\Gamma} \tau(\gamma) \sum_{k=1}^{\infty}\left|\left(\varphi_{k}, \varphi \Psi_{\gamma}\right)_{L^{2}\left(\mathbb{R}^{n}\right)}\right|^{2} d \gamma \\
= & c_{\Psi} \int_{\Gamma} \tau(\gamma)\left\|\varphi \Psi_{\gamma}\right\|_{L^{2}\left(\mathbb{R}^{n}\right)}^{2} d \gamma \\
\leq & c_{\Psi}\left(\int_{\Gamma} \frac{\tau(\gamma)}{a} d \gamma\right)\|\Psi\|_{L^{\infty}(\mathbb{R})}^{2}\|\varphi\|_{L^{2}\left(\mathbb{R}^{n}\right)}^{2}<\infty
\end{aligned}
$$

Now let $\tau$ be a complex-valued function in $L^{1}(\Gamma)$. Let $\tau=\tau_{1}+i \tau_{2}$. Write

$$
\tau_{1}^{+}-\tau_{1}^{-}
$$

and

$$
\tau_{2}^{+}-\tau_{2}^{-},
$$

where for $j=1,2$,

$$
\tau_{j}^{+}=\max \left(\tau_{j}, 0\right)
$$

and

$$
\tau_{j}^{-}=-\min \left(\tau_{j}, 0\right),
$$


we can conclude that $\varphi L_{\tau} \bar{\varphi}: L^{2}\left(\mathbb{R}^{n}\right) \rightarrow L^{2}\left(\mathbb{R}^{n}\right)$ is a trace class operator. Finally, let $\left\{\varphi_{k}: k=1,2, \ldots\right\}$ be an orthonormal basis for $L^{2}\left(\mathbb{R}^{n}\right)$. Then

$$
\begin{aligned}
\operatorname{tr}\left(\varphi L_{\tau} \bar{\varphi}\right) & =c_{\Psi} \int_{\Gamma} \tau(\gamma) \sum_{k=1}^{\infty}\left|\left(\varphi_{k}, \varphi \Psi_{\gamma}\right)_{L^{2}\left(\mathbb{R}^{n}\right)}\right|^{2} d \gamma \\
& =c_{\Psi} \int_{\Gamma} \tau(\gamma)\left\|\varphi \Psi_{\gamma}\right\|_{L^{2}\left(\mathbb{R}^{n}\right)}^{2} d \gamma
\end{aligned}
$$

as asserted.

Acknowledgements. We are grateful to the referee for the comments and suggestions on the first version of the paper.

\section{References}

[1] E. J. Candès. Ridgelets: Theory and Applications. Ph.D. Thesis, Department of Statistics, Stanford University, 1998.

[2] E. J. Candès, D. L. Donoho. Ridgelets: a key to higher-dimensional intermittency? R. Soc. Lond. Philos. Trans. Ser. A, Math. Phys. Eng. Sci., 357 (1999), 2495-2509.

[3] E. J. Candès, D. L. Donoho. Continuous curvelet transform II. discretization and frames. Appl. Comput. Harmon. Anal., 19 (2005), 198-222.

[4] V. Catană. Products of two-wavelet multipliers and their traces. in Pseudo-Differential Operators: Complex Analysis and Partial Differential Equations, Operator Theory: Advances and Applications 205, Birkhäuser, 2010, $195-211$.

[5] I. Daubechies. Time-frequency localization operators, a geometric phase space approach. IEEE Trans. Inform. Theory, 34 (1988), 605-612.

[6] I. Daubechies. Ten Lectures on Wavelets, SIAM, 1992.

[7] J. Du, M. W. Wong. Traces of wavelet multiplers. C. R. Math. Rep. Acad. Sci. Canada, 23 (2001), $148-152$.

[8] P. Goupillaud, A. Grossmann, J. Morlet. Cycle-octave and related transforms in seismic signal analysis. Geoexploration, 23 (1984), 85-102.

[9] Z. He, M. W. Wong. Wave multipliers and signals J. Austral. Math. Soc. Ser. B, 40 (1999), 437-446.

[10] H. J. Landau, H. O. Pollak. Prolate spheroidal wave functions, Fourier analysis and uncertainty. Bell Syst. Tech. J., 40 (1961), 65-84.

[11] J. Li, M. W. Wong. Localization operators for curvelet transforms. J. Pseudo-Differ. Oper. Appl., 3 (2012), $121-143$.

[12] V. B. Lidskii. Nonself-adjoint operators with trace. Dokl. Akad. Nauk SSR, 125 (1959), 485-487; Amer. Math. Soc. Translations, 47 (1961), 43-46.

[13] H. J. Pollak. Prolate spheroidal wave functions, Fourier analysis and uncertainty, III. Bell Syst. Tech. J., 41 (1962), 1295-1336.

[14] D. Slepian. On bandwidth. Proc IEEE, 64 (1976), 292-300.

[15] D. Slepian. Some comments on Fourier analysis, uncertainty and modeling. SIAM Rev., 25 (1983), $379-393$.

[16] D. Slepian, H. O. Pollak. Prolate spheroidal wave functions. Fourier analysis and uncertainty, I, Bell Syst. Tech. J., 40 (1961), 43-64.

[17] E. M. Stein, R. Shakarchi. Fourier Analysis: An Introduction. Princeton University Press, 2003.

[18] M. W. Wong. Weyl Transforms. Springer, 1998.

[19] M. W. Wong. Wavelet Transforms and Localization Operators. Birkhäuser, 2002.

[20] M. W. Wong. Discrete Fourier Analysis. Birkhäuser, 2011.

[21] M. W. Wong, Z. Zhang. Traces of two-wavelet multipliers. Integr. Equ. Oper. Theory, 42 (2002), $498-503$.

[22] M. W. Wong, Z. Zhang. Trace class norm inequalities for wavelet multipliers. Bull. London Math. Soc., 34 (2002), 739-744. 